\title{
Design and Implementation of Cold Rolling Database System in Web Data-Access System
}

\author{
Wei Teng \\ School of Electronic and Information Engineering, \\ Liaoning University of Science and Technology \\ Anshan, China \\ E-mail:tengjiawei1978@163.com
}

\author{
Guangming Wang \\ Ansteel Engineering Technology Corporation Limited \\ Anshan, China \\ E-mail: tw_ln_1978@126.com
}

\begin{abstract}
This paper describes the cold rolling database system based on Web platform and the relevant technology of this system. Adopt tree-tier B/S architecture,ASP.NET technology, C\# programming language. By communicating in different data storage of application, a dynamic, execute, interactive, highly efficient network application software has been built.
\end{abstract}

Keywords- Cold Rolling Materials; Web Database; ASP.NET; Internet

\section{INTRODUCTION}

At present, many domestic and foreign engineering material database based on Web platform have been running[1,2], but rare database completely including coldrolling steel materials, cold rolling process equipment and cold rolling process performance have been built. In this paper, we design and implement a dynamic cold rolling database system in Web data-access systems, combined with the technology of database building and accessing based on Web

$[4,5]$.

The database system is divided into the material database, cold rolling equipment database and cold rolling process database. It provides users with a standard data of the domestic cold-rolling materials, including material properties, chemical composition, physical properties, mechanical properties, microstructure properties, process parameters and process equipments. The system can provide

users with rich resources and convenient query function, can help users to select reasonable cold rolling process according to the materials, and select appropriate equipments according to the materials and process. The system improves the sharing of resources and flexibility and becomes a crossplatform and convenient resource system.

\section{INTRODUCTION OF THE SYSTEM}

\section{A. Main technical characteristics of the database system}

The database system is built on the Internet platform,we can retrieve data, increase data, modify data or delete data through the Web browser. The system connects to the SQL Server through ADO.NET of ASP.NET - a part of the Microsoft.NET, and conveniently implements database accessing by using ADO.NET components. The typical codes of SQL Server interface connection and database opening are shown below: $[6,7,8]$
SqlConnection myConnection=new SqlConnection ("server $=$ localhost $;$ uid $=$ sa ; pwd $=$ sa $;$ datebase $=$ materials");

SqlCommand cmd = new SqlCommand(" select count(*) from t_cailiao gh ", myConnection); myConnection.Open ();

The main interface of the database system is shown in Figure.1.

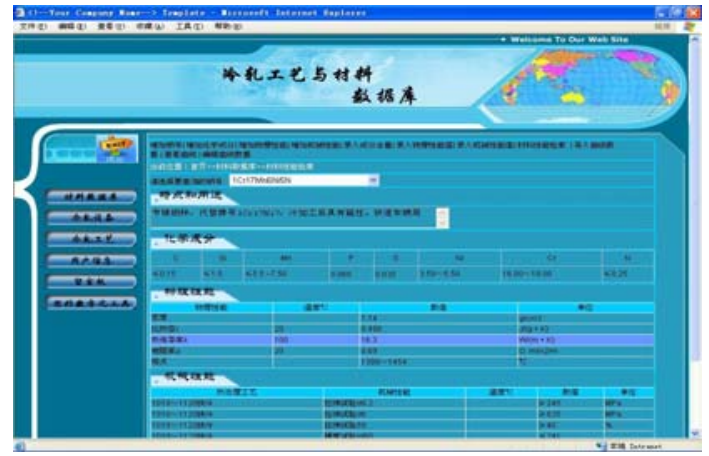

Figure 1. Database interface

1) The system uses B / S (browser / server) mode(Figure.2), separates the application module of the client from the display capabilities, and makes the module separate layer by putting it on the Web server. So that the system realizes the "thin client" as well as the isolation of user interface from business logic. The method makes maintenance and modification of the system convenient, and enhances the openness and flexibility of the system.

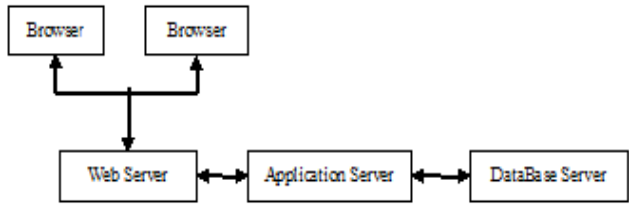

Figure 2. Tree-tier $\mathrm{B} / \mathrm{S}$ architecture

2) By using ASP.NET technology, C\# language, the database provided by server, the communication among the various data storage of application programs through ADO.NET, we establish a dynamic, interactive, efficient internet application software.

3) The clients do not need to install any software, they can browse, search, modify the data simply by using the browser (IE). 


\section{B. The software configuration of the system}

Network operating systems: server, we can select MicrosoftWindows2000 Server or Windows NT Server 4.0. the client,we can use Windows2000 or any advanced operating system.

Web server software: Internet Information Server, IIS.

Database Server: We select Structured Query Language SQL and multi-threaded relation database management system-SQL Server 2005.

The client browser: IE4.0 or any advanced browser.

\section{Features of the database system}

1) The database system has designed and implemented the user management functions, including user registration, login, user information query, modification, password finding and user right management. The system can provide different functions based on user permissions, and strengthen the security of the site.

2) Entry: By clicking on the left side of the page navigation bar--material database, device database or process database, the user can enter the database page. The administrator can complete the material database's entry of new steels (steel), chemical ingredients, physical properties, mechanical properties and the entry of chemical composition and contents, mechanical properties and parameter values, physical properties and parameter values of each steel. The administrator can enter the type of equipments (such as pickling equipments or rolling equipments), device name, the main performance and the corresponding device pictures into the device database. The administrator also can complete the process database's entry of process parameters based on all kinds of processes.

3) Retrieval: By clicking the button to enter the corresponding page of the left-hand navigation bar, users can retrieve detailed information based on retrieved steel (including the physical properties, mechanical properties, chemical composition, and steel basic information), the basic information of cold rolling equipments at a variety of ways (through category and name), the cold rolling process parameters of specified products based on steel.

4) Modification, deletion: The authorized user can modify and delete data while he is entering data.

5) Chart information processing: The cold rolling material microstructure and properties, mechanical properties and process performance are usually described by chart. The paper designs and implements chart processing functions, that put chart information into digital information and import digital information into material database and redraw curves and the corresponding information in order that the user can retrieve and browse data visually.

\section{DATABASE STRUCTURE} [9].

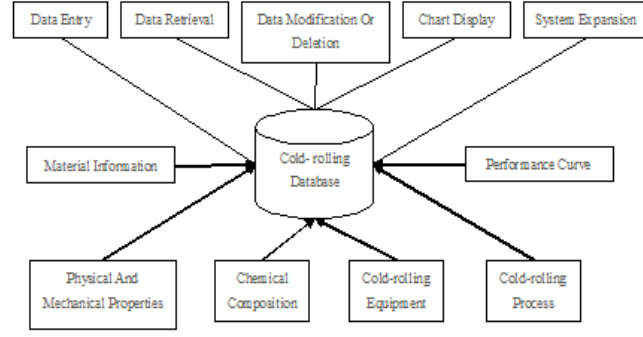

Figure 3. Database structure

1) "The material information" has stored domestic cold rolling steel's brand, category, main characteristics, main useness, chemical composition, physical properties( including the density under typical temperature, specific heat, coefficient of linear thermal expansion, thermal conductivity and other data), mechanical properties ( including the mechanical properties under given heat treatment system, fatigue properties data ). Each datatable is given by independent subtable.

2) The " physical and mechanical properties " refer to that the physical and mechanical performance parameters of cold rolling material are stored in respective data table.

3) The "cold rolling equipment" includes the category table of cold rolling equipment and the table of cold rolling equipment. The former stores information of cold rolling equipment classification, the latter stores equipment description, equipment detailed information and equipment pictures according to the classification of corresponding device.

4) The "performance curve" stores the two-dimensional data of the curves, the number of the marking data, the detailed information of the chart (such as the material hardness in different cooling rates and the material microstructure changes under the different cooling temperature range of CCT curves.).

5) The "Cold-rolling Process" records the corresponding process parameters according to the different processes (e.g., pickling, rolling, annealing, formation).

\section{THE KEY TECHNOLOGY OF THE DATABASE-THE DESIGNMENT OF GRAPHIC PROCESSING TOOLS}

1) User requirements: We should put the graphs of cold rolling material microstructure and mechanical properties recorded in the books, journals, magazines and other written documentation into electronic curves, then convert the curves into two-dimensional data and store data, so that the data can be permanently retained, updated and shared conveniently.

2) Design: The graphics are scanned into the computer and converted into graphic files (such as JPG, BMP etc.). According to the graphics we can select automatic or manual method of location selection. Then, corresponding data and information of the graphics are stored into a EXCEL file, which is imported into the database. Fitting the curve on Web (the interpolation algorithm) and displaying curves' information.

3) Data flow diagram (Figure.4): The procedures are described in the data flow diagram [10], we design 
corresponding modules and the module codes according to each part of the function.

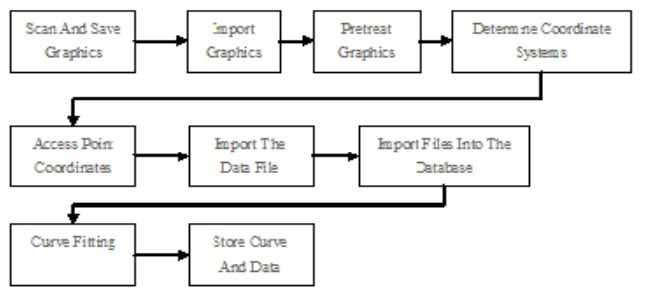

Figure 4. The flowchart of graph tool

4) Test: The test data and the test method are designed. Each module and the network should be tested.

5) Example: SCr440 CCT curve fitting interface is shown below (Figure.5)

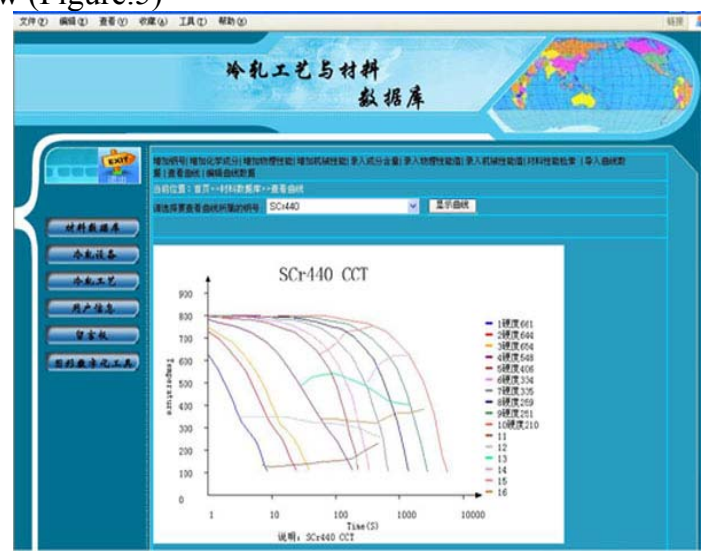

Figure 5. The interface of curve fitting

\section{CONCLUSIONS}

In this paper, we have built a cold rolling database system based on the B / S three layer network structure by using ASP.NET technology. The system have reflected the advantage of ASP.NET technology. Only through IE, users can complete all information query and management. The database have a large number of accurate data, add the curve processing function, make up of the blank field of cold rolling material and process database among material database. While there are still many shortcomings in this system. We will continue to improve and extend the function based on user's needs, and strengthen the management of authority of the system. At the same time, we will increase the functions of data analysis and data mining to establish a more intelligent database system.

\section{REFERENCES}

[1] GU Qiang, Zhong Rui, JU Dong-ying, "Development of Materials Database System for CAE System of Heat Treatment Based on Data Mining Technology", Transaction of Nonferrous Metals Society of China, Vol.16, pp.572, 2006

[2] T. Inoue and K. Okamura, D.Y. JU, "Material Database for Simulation of Metallo-thermo-mechanical Fields", Proceedings of 20th ASM Heat Treating Conference and 5th International Symposium on Quenching and Distortion Control, ASM International, St Louis, pp.753, October 2000

[3] Li Chunsheng, Huang Debin, Metal Materials handbook(II). Chemical Industry Press, 2005

[4] Fu Jian, Li Shuping, ZhangGuangming," Study on key techniques to design the database structure of mould material, thermo-process plan and performance",Moden Manufacturing Engineering, Vol.8, pp.4647, 2006

[5] Xia Qing,Yin Guofu, Hu Xiaobing, Wang Jianfei," Development of engineering material database system on web",Machinery, Vol.32, pp.55-57, 2005

[6] Wang Hanzhu, Yang Yanze, Wang Li, Wang Shu," Development of Inorganic-Nonmetallic Material Database on Web",.Materials Review, Vol.16, pp.75-76, 2002

[7] Zhang Ping, Xie Qiang, Ding Qiulin,'The General Method of Accessing to Database Based on ASP.NET", Journal Of Computer Applications, Vol..24, pp.256-257, 2004

[8] Yu Qian, Luo Jing, "The Database Management System By ADO.NET", Microcomputer Application, Vol..25, pp.373-375, 2004.

[9] Bai Jianyu, Xu Jinfu, Bao Mingdong,"'The Database of Cold-punched Die Material and Its Application" ,Materials for Mechanical Engineering, Vol.25, pp.36-39, 2001

[10] Tong Xiaoyan,'The Design of User-Oriented Database of Material Performance Based on Client/Server Model", Technical Proceedings of the Materials Science and Engineering, pp.259-264, 1998. 